Man and Nature

MAN AND NATURE

L'homme et la nature

L'HOMME ET LA NATURE

\title{
À la recherche du malade dans Le Mariage de Figaro : quel(s) sens faut-il donner à l'objet-fauteuil?
}

\section{Georges-L. Bérubé}

Volume 5, 1986

URI : https://id.erudit.org/iderudit/1011849ar

DOI : https://doi.org/10.7202/1011849ar

Aller au sommaire du numéro

Éditeur(s)

Canadian Society for Eighteenth-Century Studies / Société canadienne d'étude du dix-huitième siècle

ISSN

0824-3298 (imprimé)

1927-8810 (numérique)

Découvrir la revue

Citer cet article

Bérubé, G.-L. (1986). À la recherche du malade dans Le Mariage de Figaro : quel(s) sens faut-il donner à l'objet-fauteuil? Man and Nature / L'homme et la nature, 5, 15-28. https://doi.org/10.7202/1011849ar

Copyright (c) Canadian Society for Eighteenth-Century Studies / Sociéte canadienne d'étude du dix-huitième siècle, 1986
Ce document est protégé par la loi sur le droit d'auteur. L'utilisation des services d'Érudit (y compris la reproduction) est assujettie à sa politique d'utilisation que vous pouvez consulter en ligne.

https://apropos.erudit.org/fr/usagers/politique-dutilisation/ 


\section{A la recherche du malade dans Le Mariage de Figaro: quel(s) sens faut-il donner à l'objet-fauteuil?}

Dans la didascalie initiale du Mariage de Figaro, Beaumarchais indique le décor où va se dérouler l'action du premier acte de la pièce: 'Le théâtre représente une chambre à demi démeublée; un grand fauteuil de malade est au milieu. ${ }^{\prime}$ Cette chambre, dépouillée à l'excès - d'une 'nudité exceptionnelle dans le théâtre du temps' ${ }^{\prime 2}$ selon Jacques Scherer - doit servir de chambre nuptiale à Suzanne et à Figaro: tel est du moins le projet du Comte. Ainsi, c'est un Figaro visiblement satisfait de son sort qui s'affaire dès le lever du rideau à mesurer le plancher de l'appartement, pour voir, dit-il, 'si ce beau lit que monseigneur nous donne aura bonne grâce ici. $^{\prime 3} \mathrm{Vu}$ la situation initiale dans laquelle se trouvent les deux personnages, ainsi que la nature du décor, le spectateur/lecteur se serait sans doute attendu à voir des objets qui cadrent mieux avec la chambre nuptiale: un lit, par exemple celui que le Comte Almaviva entend donner en cadeau aux fiancés; or, tout ce qu'on lui donne à voir, c'est un grand fauteuil de malade. Objet, par conséquent, insolite dans un tel décor, objet qui intrigue le spectateur et qui fait dire à Jean-Michel Adam que 'l'intérêt principal du fauteuil de malade est son "étrangeté" par rapport au lieu référentiel où il se trouve. ${ }^{4}$ En effet, tous ceux qui assistent au début de l'action ne sauraient tarder à se poser, de façon plus ou moins consciente, les questions suivantes: que vient faire dans une pièce prétendument réservée à l'amour le grand fauteuil de malade? Et, puisqu'il est là, 
où se trouve le malade qui doit nécessairement l'occuper? Dans les pages qui suivent, nous avons tenté de réconcilier l'objet et son décor, ou du moins de montrer que leur présence ensemble - si paradoxale soit-elle peut avoir un/des sens. Autrement dit, nous nous sommes mis à la recherche du malade dans le Mariage de Figaro.

Jacques Scherer, dans son analyse dramaturgique des premières scènes de la pièce, passe sous silence ce gros objet pour le moins gênant, apparemment convaincu que l'explication qu'il en donne dans son introduction à l'acte I est suffisante:

la chambre destinée à Figaro et à Suzanne ... est à peu près vide, sans doute parce que les meubles ont été déplacés pour permettre l'aménagement de la pièce qu'occuperont les futurs époux .... Seul, au milieu de la scène, trône un vaste fauteuil de malade: comme nul n'est malade dans la comédie, on doit penser que ce fauteuil peu utile a été laissé là parce qu'il était trop encombrant et qu'on ne savait où le mettre. ${ }^{5}$

Etant donné la perspective adoptée par J. Scherer, c'est-à-dire celle qui consiste à montrer l'utilité des objets sur la scène, nous devons être d'accord avec lui: à ce moment-ci de l'action, le fauteuil ne sert à rien. Quant aux raisons invoquées pour expliquer sa présence dans la pièce, elles sont aussi peu probantes les unes que les autres. Quoi qu'il en soit, Scherer n'a pas dit là son dernier mot puisqu'il entreprend à partir de l'entrée de Chérubin (A. I, sc.7) une étude nuancée et fort révélatrice des divers rôles que joue le fauteuil de malade: obstacle physique, écran à deux puis à trois dimensions, simple fauteuil, etc. L'intérêt et la richesse même de cette étude rendent alors les premières raisons d'autant plus problématiques. Veut-il nous faire croire que ce 'vaste fauteuil' tire son sens de sa seule utilité, des seuls rôles que lui font jouer les personnages sur la scène? et qu'auparavant - donc des scènes 1 à 6 - on doit $y$ voir un objet inutile, voire superflu? Un objet, somme toute vide comme le lieu, qui serait en attente de son sens? L'explication, pour plausible qu'elle soit dans le théâtre moderne, est peu probable à l'époque de Beaumarchais.

Jacques Scherer n'est pourtant pas le seul à considérer le fauteuil comme peu utile avant l'entrée de Chérubin. Jean-Michel Adam propose une lecture sémiologique qui dégage la même conclusion:

C'est d'abord un objet sans fonction: fauteuil de malade, il n'est occupé par aucun malade. Rarement considéré comme un siège, cet objet acquiert progressivement du sens; un contenu lui est conféré par les personnages qui l'utilisent et occupent différentes places par rapport à lui. ${ }^{6}$ 
Et Adam ajoute en guise d'explication:

Initialement, comme il n'est occupé par aucun malade et qu'il se trouve dans la chambre de futurs mariés, c'est un signe vide. Son signifié utilitaire (sa "transparence" J.-F. Lyotard) étant retenu, il doit acquérir une progressive épaisseur ("opacité") sémantique par sa mise en circulation dans le système scénique. $^{7}$

'Un signe vide,' nous dit J.-M. Adam jusqu'au moment du jeu de poursuite entre Chérubin et Suzanne. Mais qu'est-ce qu'un signe vide? Parce que le fauteuil n'est occupé par aucun malade, est-ce à dire qu'on ne retient de lui que sa transparence et, donc, son inutilité? Cette interprétation nous paraît d'autant moins satisfaisante que Beaumarchais porte une attention toute spéciale au dispositif scénique: aux acteurs, il s'efforce de recommander au début de son texte qu'ils conservent 'les bonnes positions théâtrales.' Ainsi, placer un fauteuil aussi encombrant au centre du plateau et ce, pendant six scènes du premier acte, pour se contenter de sa seule inutilité, voilà qui n'étonnerait pas peu de la part du dramaturge minutieux que fut Beaumarchais. ${ }^{8}$ D'ailleurs, n'oublions pas que le fauteuil continue d'occuper sa position centrale même après les scènes où on l'utilise de façon explicite: par exemple à la scène dix quand le Comte remet la toque de la mariée à Suzanne devant tous les personnages réunis. Serait-ce que Beaumarchais s'est montré singulièrement malhabile de ne pas avoir introduit le fauteuil juste avant son utilisation et de ne pas l'avoir fait disparaître juste après? Nous reviendrons à cette question plus tard.

Guy Michaud, dans l'Oeuvre et ses techniques, exprime des idées semblables:

A ce moment (l'arrivée de Chérubin) entre en jeu un autre personnage, présent depuis le lever du rideau, mais auquel, bien qu'il fût "au milieu de la scène," le spectateur n'avait guère prêté attention jusque-là. Je veux parler du fauteuil ${ }^{9}$ (souligné par l'auteur).

Michaud conclut avec cette remarque pertinente:

Si précisément l'auteur lui a assigné cette position centrale, on se doute que ce n'est pas pour rien. C'est en effet autour du fauteuil que Chérubin va tourner et faire tourner Suzanne après lui. ${ }^{10}$

Selon nous, Michaud a tout à fait raison de souligner l'importance scénique du fauteuil, mais pourquoi retarde-t-il son entrée en jeu jusqu'à la scène sept? 
Récapitulons: avant l'arrivée de Chérubin, le fauteuil est un 'objet peu utile' (Scherer); un 'objet sans fonction' et 'un signe vide' (Adam); c'est un 'personnage' (ou force dramatique dans le sens de Souriau), présent depuis le début, mais non agissant (Michaud). Cette interpétation, unanime semble-t-il, ne laisse pas de créer certains problèmes, car elle repose, selon nous, sur une hypothèse qui est loin d'être prouvée, à savoir qu'il n'y a en réalité aucun malade au début de la pièce et, donc, que le fauteuil de malade n'y trouve pas sa raison d'être.

Dans la chambre des futurs mariés, il n'y a aucun malade - voilà l'évidence même selon nos trois auteurs - et nous sommes d'accord, si la notion de malade se limite à un personnage présent et jouant ce rôle sur la scène. Beaumarchais prend d'ailleurs un malin plaisir à confirmer cette évidence: à la scène quatre, Bartholo demande à Marceline pourquoi elle l'a fait venir, lui un médecin, au château:

BARTHOLO: $\quad$ Eh bien, qui rend donc ma présence au château si nécessaire? Monsieur le Comte a-t-il eu quelque accident?

MARCELINE: Non, docteur.

BARTHOLO: La Rosine, sa trompeuse Comtesse, est-elle incommodée, Dieu merci?

MARCELINE: $\quad$ Elle languit.

BARTHOLO: $\quad$ Et de quoi?

MARCELINE: $\quad$ Son mari la néglige.

BARTHOLO: $\quad$ (avec joie) Ah! le digne époux qui me venge. ${ }^{11}$

La situation pénible de la Comtesse ne constitue pas le genre de maladie qui nécessite un grand fauteuil de malade. Languir, c'est un peu comme avoir des vapeurs, une faiblesse temporaire réservée aux femmes du monde: 'un mal de condition, explique Suzanne, qu'on ne prend que dans les boudoirs.' ${ }^{\prime 2}$ Non, reprend Marceline, il n'y a pas de malade; Bartholo n'a pas été appelé en sa qualité de médecin, mais de vieil ami. Le spectateur conclut donc: tout ce qui a trait à la médecine est un leurre; médecin, malade, fauteuil, des artifices hérités de la comédie traditionnelle. Et pourtant, tant d'allusions piquent la curiosité ... 
Songeons à la situation initiale de la pièce: Figaro accepte avec une joyeuse innocence la chambre et le lit que le Comte lui donne à l'occasion de son mariage; Suzanne, elle, s'oppose à ces 'cadeaux' suspects pour des raisons qu'elle refuse de révéler pour l'instant. Elle fait comprendre à Figaro néanmoins qu'il s'agit d'un cadeau grec dont il faut se méfier. Si la soubrette avait pu citer Virgile, elle aurait sûrement repris ces mots célèbres de l'Enéide: 'Timeo Danaos et dona ferentes.' En fait, accepter la chambre et le lit de Monseigneur c'était permettre au loup d'entrer dans la bergerie ou, ce qui revient au même, accueillir l'amant dans le but de faire un ménage à trois. Plus concrètement encore, accepter cette chambre, c'était aussi accepter de vivre avec le grand fauteuil de malade - sans jamais pouvoir s'en débarrasser. Voilà à quoi le naïf Figaro consent de prime abord: d'après ses calculs, le lit et le fauteuil peuvent entrer tous les deux dans la chambre sans problèmes, ils peuvent coexister en faisant bon ménage. Suzanne, plus lucide, refuse et le lit, et la chambre, et le fauteuil de malade. Elle ne s'arrête pas pour se demander si le fauteuil est utile, s'il est insolite; le fauteuil s'impose à elle d'emblée comme un obstacle ou une menace qu'il faut écarter. Ainsi, c'est dans les rapports qui s'établissent entre l'objet et les personnages que se trouve la clé de son/ses sens. Plutôt que d'insister avec Scherer et les autres sur le fait que le fauteuil est vide, qu'il ne sert à rien, nous aimerions faire remarquer qu'il est là, que cet 'être-là' n'est pas, quant à lui, vide de sens.

Le metteur en scène qui se propose de monter le Mariage de Figaro est sans doute celui qui a la meilleure saisie du premier sens qu'il faut donner au fauteuil de malade. Pourquoi? Parce qu'il doit composer constamment avec ce gros objet accaparant. Jean Meyer, dans sa mise en scène publiée au Seuil, indique toujours la position des comédiens par rapport à l'objet: quand Figaro et Suzanne évoquent leur bonheur futur, Meyer exige qu'ils s'éloignent le plus possible du fauteuil, ou qu'ils le contournent avec attention. Lorsqu'au contraire, Suzanne fait allusion aux obstacles à surmonter, elle se rapproche insensiblement de lui. 'Tout en parlant, (Suzanne) vient s'asseoir sur le bras droit du fauteuil'13 écrit J. Meyer, pour révéler à Figaro les prétentions du Comte à exercer son droit du seigneur. Comprenons donc que le fauteuil signifie dès le départ l'obstacle: c'est une masse qu'il faut tenter de contourner, d'éviter, mais une masse imposante qui domine la pièce. Le premier sens à donner au fauteuil pourrait s'énoncer ainsi: un obstacle qui vaut d'abord par sa seule matérialité. Sa présence dans la chambre nuptiale entrave les mouvements des personnages avant de nuire à leur bien-être, ce qui revient à dire que son rôle à ce moment-ci de l'action est purement négatif: d'être nuisible.

Les personnages ne tardent toutefois pas à donner un contenu positif 
à l'obstacle, pour que le spectateur comprenne très rapidement le paradigme à l'œuvre ici. En quoi consiste l'obstable pour Suzanne? En la prétention du Comte à exercer son ancien droit du seigneur. S'établit alors un lien, vaguement suggéré au départ mais qui se précise de plus en plus, entre l'obstacle (le fauteuil) et le Comte. A ce sujet, il est intéressant de rappeler les indications de Meyer pour la scène quatre de l'acte I: Marceline et Bartholo 'se trouvent de part et d'autre du fauteuil'14 lorsqu'ils peignent en deux mots le portrait du Comte: 'il est jaloux et libertin. ${ }^{\prime 15}$

Le lien ténu entre le Comte et le fauteuil explique en quelque sorte pourquoi à la scène 1 Suzanne le rejette de façon aussi catégorique. ${ }^{16}$ De plus, nous comprenons la raison pour laquelle le fauteuil continue à dominer la scène, à l'habiter de son ombre pendant le monologue de Figaro (A.I, sc.2). En effet, celui-ci s'en prend au Comte qui veut l'obliger à jouer le rôle du mari cocu; il l'interpelle sur un ton vindicatif: 'J'entends monsieur le Comte: trois promotions à la fois: vous, compagnon ministre, moi, casse-cou politique, et Suzon, dame du lieu, l'ambassadrice de poche, et puis, fouette courrier! Pendant que je galoperais d'un côté, vous feriez faire de l'autre à ma belle un joli chemin. ${ }^{17}$ Le seul interlocuteur de Figaro, c'est le vaste fauteuil muet: obstacle immuable auquel Figaro doit faire face, contre lequel il lutte sans jamais pouvoir y échapper.

Aux scènes quatre et cinq, Marceline dévoile son projet d'épouser Figaro avec l'aide du Comte; en présence du fauteuil et comme si elle le prenait à témoin, elle assure à Suzanne que son mariage n'est pas chose certaine et qu'elle, Marceline, réussira à lui enlever son fiancé. Si l'on se souvient finalement des problèmes de ménage qu'éprouvent le Comte et la Comtesse, on s'aperçoit que le fauteuil de malade a été omniprésent dans l'exposition, et qu'il en fait, sans aucun doute, partie intégrante. En réalité, l'élément-clef dans tous ces conflits, le principal obstacle, c'est le Comte - celui-là même qui détient le pouvoir de les causer, comme de les résoudre, et le seul qui par là a droit à un fauteuil.

Posons à nouveau la question du début: y a-t-il un malade dans cette œuvre? La réponse, bien que toujours négative en apparence, doit être nuancée: à défaut d'un malade, nous avons identifié un objet qui représente un personnage souffrant d'un mal chronique, pour lequel il n'existe aucun remède... 'Jaloux et libertin': voilà le mal moral dont ce grand d'Espagne est atteint. Plutôt que de s'occuper de politique internationale et de haute justice, il s'amuse à faire la conquête des paysannes (Fanchette), à affirmer son pouvoir abusif et tyrannique sur les soubrettes (Suzanne), tout en soupçonnant sa propre femme de trahison. Singulière destinée pour le pouvoir d'être réduit à la simple intrigue 
amoureuse. ${ }^{18}$ Le grand fauteuil de malade fonctionne ici comme le signe visible d'une vérité cachée: dans la mesure où le fauteuil est le symbole du Comte et de son pouvoir, il dit l'état lamentable dans lequel ce pouvoir est tombé. Bref, le fauteuil appartient en propre au Comte - il sera d'ailleurs le seul à s'y asseoir si nous exceptons Chérubin qui s'y cache un bref instant - et symbolise le pouvoir au même titre qu'une couronne ou un véritable trône pourraient le faire. En revanche, en en faisant un fauteuil de malade, Beaumarchais érige, en un effet de parodie visuelle, une ironique caricature de trône qui donne le ton à toute la comédie. Christiane Mervaud a judicieusement remarqué, à propos du ruban de nuit de la Comtesse, que l'accessoire (dans le théâtre de Beaumarchais) n'est plus l'adjuvant d'un dialogue dont il rythme la progression, un relais pour que puisse s'épanouir le discours roi, mais langage à part entière, disant ce qui, sans lui, ne pourrait être dit et l'exprimant dramatiquement. ${ }^{19}$ Sans aller jusqu'à faire exprimer par ses protagonistes la 'maladie' du pouvoir, Beaumarchais parvient malgré tout à la signifier par ce nouveau 'langage' que constitue pour lui l'objet théâtral.

Le fauteuil dans les premières scènes, d'inutile qu'il était, devient dans cette perspective un signe aux connotations multiples. En fait, il annonce clairement la défaite d'un Comte lucide, rusé et formé à l'école de la diplomatie, et qui pourtant sera amené à avouer pitoyablement dans son monologue à l'acte trois: 'le fil m'échappe' et, plus tard, en aparté: 'il y a un mauvais génie qui tourne tout ici contre moi!'20 Le pouvoir étant malade, les sujets peuvent se permettre toutes sortes de libertés, que ce soient les intrigues de Figaro, celles des femmes ou les fredaines de Chérubin. Mais en quoi consiste cette supposée maladie du trône? Quels en sont les symptômes?

Les rôles dramaturgiques dégagés par J. Scherer illustrent concrètement la nature et l'étendue de ce mal. D'abord, écrit le critique, 'le grand fauteuil sert d'obstacle, autour duquel tournent les deux coureurs. ${ }^{21}$ Rappelons que Chérubin, épris de la Comtesse, arrache à Suzanne le ruban de la bien-aimée, ce qui donne lieu à un jeu de poursuite dans lequel le fauteuil sert d'obstacle. Au strict niveau dramaturgique, cette interprétation rend bien compte du rôle que joue le fauteuil sur la scène. Et J.M. Adam a raison d'affirmer que le signe /fauteuil/ va 'acquérir une progressive épaisseur ("opacité") sémantique par sa mise en circulation dans le système scénique.' Par contre, Adam a tort selon nous de retarder cette implication de l'objet jusqu' à l'entrée de Chérubin, puisque, comme nous avons tenté de le montrer, son fonctionnement sémantique commence dès les premières scènes dans la mesure où il y est perçu comme objetobstacle riche en connotations. De ce fait, le fauteuil de malade autour duquel tournent les deux jeunes protagonistes n'est plus objet vide de 
sens, ni même simple obstacle physique, il est déjà le signe du trône: un trône, on le sent bien, qui désigne son immobilité, sa paralysie et son impuissance. Le vol du ruban et le jeu de poursuite perdent dans ce contexte leur caractère ludique, voire innocent, pour devenir de véritables actes coupables, des crimes de lèse-majesté: Chérubin, le braconnier, chasse sur les terres réservées au Comte - on ne fait jamais la cour à la femme et à la maîtresse désignée d'un gentilhomme espagnol sans s'exposer à de graves sanctions; mais le Comte (son trône) n'est déjà plus qu'un obstacle qu'on contourne en folâtrant. La lecture ici est double: l'obstacle, de physique qu'il était au niveau dramaturgique, devient à un niveau sémantique plus profond un obstacle moral, social et politique. Chérubin, en se moquant du mari, de l'aristocrate et du maître du domaine, viole par son jeu des droits sacrés: il remet en cause les privilèges traditionnels de l'autorité absolue. Et - voilà qui est essentiel - il le fait impunément. Peut-on mettre en lumière de façon plus frappante la faiblesse du trône et la maladie chronique de celui qui l'occupe?

Deuxième rôle, selon J. Scherer, 'le fauteuil ... devient un écran: il n'est plus ce qui empêche de saisir la personne qu'on voit, il est ce qui dissimule la personne.'22 Rappelons que, dans la pièce, le jeu de poursuite est bientôt interrompu par l'arrivée inopinée du Comte, ce qui oblige Chérubin à chercher refuge derrière le fauteuil, seule cachette disponible à ce moment-là. L'écran, que J. Scherer analyse avec beaucoup de finesse, n'est toutefois pas simple écran; en réalité, Chérubin se camoufle derrière un écran-fauteuil, derrière le trône qui voile l'intrus aux yeux mêmes de son maître. A l'impuissance connotée par le fauteuil-obstacle, nous ajoutons une nouvelle caractéristique à la maladie d'Almaviva, celle connotée par le fauteuil-écran: la cécité. Voilà qui deviendra péniblement évident au moment où le Comte, ignorant la situation réelle, prend le fauteuil de malade pour ce qu'il pourrait être: un simple fauteuil. C'est donc assis, comme le veut la coutume, que le maître va tenter de séduire Suzanne, sans soupçonner qu'il est en train de révéler à son rival un projet secret. Plus tard, quand le Comte découvre - tout à fait par hasard, il va sans dire - la cachette du page, il se montre incapable de percer les ténèbres autour de lui: on doit tout lui expliquer.

SUZANNE: Votre arrivée l'a si fort troublé qu'il (Chérubin) s'est masqué de ce fauteuil.

LE COMTE

(en colère):

Ruse d'enfer! Je m'y suis assis en entrant.

CHÉRUBIN:

Hélas, monseigneur, j'étais tremblant derrière. 
LE COMTE: $\quad$ Autre fourberie! Je viens de m'y placer moi-même.

CHÉRUBIN: $\quad$ Pardon; mais c'est alors que je me suis blotti dedans. ${ }^{23}$

Privé de la lucidité qui doit caractériser le pouvoir, le Comte s'assied sur son trône croyant béatement en son pouvoir absolu; pourtant, il devra par la suite - c'est là le comble de l'ironie - se servir de son propre trône comme écran pour tenter de surprendre la vérité. ${ }^{24}$ Ces manœuvres s'avèrent vaines malgré tout, puisque le Comte n'a pas plutôt quitté son siège pour passer derrière l'écran que Chérubin se faufile devant pour se blottir dans le fauteuil, à la place même de son maître. L'usurpation, qui n'était que partielle lors du rapt du ruban, ${ }^{25}$ devient totale, de sorte que le page pourra désormais remplacer le Comte auprès de la Comtesse, entreprendre de la séduire, et ensuite - cela nous est narré dans la Mère coupable - lui faire un enfant. En somme, le fauteuil-écran est le signe même de la cécité du trône; le Comte aimerait bien exploiter l'écran comme Chérubin l'avait fait avant lui, mais autant celui-ci réussit, autant celui-là échoue. Ne devient pas clairvoyant qui veut!

Le grand fauteuil joue enfin un troisième rôle dans cette pièce: Suzanne, qui pense à sa réputation compromise, voit tout à coup surgir le Comte de sa cachette; elle feint alors de se sentir mal: 'Elle est saisie, dit le Comte à Bazile. Asseyons-la dans ce fauteuil.'26 Pour la première fois, le fauteuil de malade pourrait accueillir un personnage 'malade,' mais Suzanne, effrayée par l'effet néfaste que produirait la découverte de Chérubin, refuse de s'y asseoir. Cela signifie, nous semble-t-il, que le fauteuil de malade ne peut pas jouer son rôle premier, qu'il n'est pas là comme signe transparent, mais plutôt, dans ce cas, pour recevoir Chérubin. Parlons donc d'un fauteuil-repaire qui offre aux plus habiles une niche au coeur du pouvoir. Le Comte 'outré' s'exclame alors: 'C'est donc une couleuvre que ce petit ... serpent-là!'27 A son insu, le Comte a nourri dans son sein une couleuvre, non - le mot n'est pas assez fort un serpent, qui ne manquera pas de jouer plus tard le rôle mythique du séducteur de la femme. Ainsi le trône est miné de l'intérieur par un mal qu'on a soigneusement cultivé: le fauteuil-repaire révèle un pouvoir affaibli dont le coeur même est atteint.

Après cet épisode, J. Scherer conclut: 'Le fauteuil a rempli son rôle parfaitement, explication comprise. Chérubin peut en sortir. Désormais inutile, l'immense fauteuil de malade sera comme invisible pour le public. ${ }^{28}$ Toujours le même, l'argument consiste à dire que le fauteuil, ne servant plus aux personnages, devient ipso facto inutile, donc 'comme invisible' jusqu'à la fin de l'acte. Mais justement, la fin de l'acte I contient la scène de groupe où toute la collectivité prie le Comte de renoncer of- 
ficiellement à son droit du seigneur en remettant à Suzanne la toque virginale. Dans cette scène de revendication sociale collective, ${ }^{29}$ où le spectacle est recherché pour lui-même - Beaumarchais aimait les tableaux émouvants à la Diderot - , le fauteuil, loin d'être invisible, occupe toujours le centre du plateau sans que rien ni personne ne puisse le déloger. Selon nous, son sens saute aux yeux si l'on accepte l'interprétation avancée ici: face à son peuple, à ses rivaux (Chérubin, Figaro), à sa femme, le Comte est contraint à céder; 'il est piquant, écrit Scherer, qu'il soit obligé d'accepter ce qui est le plus contraire à ce qu'il désire vraiment. ${ }^{30}$ Piquant, oui, mais étonnant, non; depuis le début, Beaumarchais nous a donné à entendre par le langage de l'objet que le pouvoir était aveugle, impuissant, miné de l'intérieur, qu'on pouvait le manipuler à condition de savoir comment s'y prendre. La réunion sur la scène du Comte et du fauteuil au moment même où leur défaite est consacrée rend d'autant plus explicite le lien qui les unit: le fauteuil est le signe du pouvoir du Comte - s'il est vaste, il est malade.

Par ailleurs, Beaumarchais n'abandonne pas à la fin de l'acte I un objet aussi signifiant, puisqu'il revient à la charge à l'acte III, celui du procès ou de la 'comédie judiciaire' (Scherer), en mettant de nouveau en scène un fauteuil. La didascalie initiale précise: 'le théâtre représente une salle du château appelée salle du trône, servant de salle d'audience, ayant sur le côté une impériale en dais, et dessous, le portrait du roi. ${ }^{31}$ Dans cette 'salle du trône,' ne manque pas de se trouver le symbole du pouvoir, comme l'indique Figaro au Comte qui lui demande si tout est prêt pour l'audience publique: 'Le grand fauteuil pour vous, de bonnes chaises aux prud'hommes, le tabouret du greffier, deux banquettes aux avocats, le plancher pour le beau monde et la canaille derrière. ${ }^{32}$ Ainsi, lorsque l'audience commence à la scène quinze, nous lisons: 'Le Comte s'assied sur le grand fauteuil.'33 Beaumarchais se garde bien ici de qualifier ce fauteuil de fauteuil de malade; l'allusion trop explicite aurait presque sûrement entraîné des sanctions sévères de la part des autorités. Le spectateur, en revanche, n'est pas dupe, il saisit très bien que ce fauteuil-trône de l'acte III doit être compris à la lumière du paradigme construit à l'acte I: fauteuil de malade-trône. En outre, Figaro se charge de montrer qu'à son époque un procès n'a rien à voir avec la justice, qu'en matière de procès forme et fond sont tout à fait distincts: Monsieur, dit-il au juge Brid'oison, je m'en rapporte à votre équité, quoique vous soyez de notre justice. ${ }^{34}$ Mais quoi que fasse le vaillant Figaro, le Comte (le trône) a déjà décidé de sa culpabilité. Si l'acte du procès illustre clairement les abus inhérents au système judiciaire, Beaumarchais avait déjà inscrit en filigrane à l'acte I la nature même du pouvoir, grâce à l'objet insolite qu'est le grand fauteuil de malade. 
Le spectateur pourrait croire qu'il en a maintenant fini avec le fauteuil, mais tel n'est pas le cas. Beaumarchais, peu disposé à se défaire d'un objet si polyvalent, lui fait subir une métamorphose de sorte qu'à l'acte $\mathrm{V}$ nous le retrouvons sous la forme bien primitive d'un siège de gazon. De l'avis de J. Scherer, le décor de cet acte représente le lieu d'un jugement', où la clairière est une 'version champêtre du prétoire' avec 'au centre un siège de gazon pour l'accusé. C'est un tribunal de verdure. ${ }^{35} \mathrm{De}$ juge qu'il était, le Comte devient l'accusé qui sera ensuite condamné par le tribunal populaire. N'est-ce pas là la preuve, ou l'éclatante confirmation, de la maladie du Comte et du trône? A l'extérieur du château d'Aguas-Frescas, où la nature reprend ses droits, on assiste au dévoilement public de l'état de santé du Comte: 'jaloux et libertin.'

Cette étude aura contribué, on l'espère, à persuader que le théâtre tolère rarement un objet purement 'inutile'; et que c'est un contresens, selon nous, de nier l'utilité d'un objet sous prétexte qu'il ne sert pas concrètement aux personnages. Le grand fauteuil de malade, s'il ne sert pas de siège au début de la pièce, et a donc perdu, contrairement à ce que pense J.-M. Adam, son signifié utilitaire ou sa 'transparence,' n'est pas inutile pour autant; il sert, dès l'entrée en scène des personnages, à signifier par sa matérialité l'obstacle nuisible qu'il constituera pendant tout le premier acte. Dès lors, on arrive à établir le lien entre l'obstacle et le Comte: le fauteuil de malade est le signe du Comte comme la couronne peut être le signe conventionnel du pouvoir. D'ailleurs, comment expliquer que l'auteur ait voulu un grand fauteuil de malade sinon par son intention de signifier le trône, le grand siège sur lequel s'assoit le pouvoir absolu. L'audace de Beaumarchais avait de quoi étonner ses contemporains: la parodie du procès à l'acte III se déroule sous le portrait du roi. Cette audace paraît plus étonnante encore quand, grâce au paradigme de l'acte I, on comprend que le fauteuil de malade et le fauteuil du trône ne font plus qu'un.

GEORGES-L. BÉRUBÉ

Université York 


\section{NOTES}

1 Beaumarchais, Théâtre, éd. de M. Rat (Paris: Garnier 1964) 179. Toutes les références au texte renvoient à cette édition.

2 Beaumarchais, le Mariage de Figaro, éd. avec analyse dramaturgique par Jacques Scherer (Paris: S.E.D.E.S. 1966) 51

3 A.I, sc. 1, p. 179

4 Jean-Michel Adam et Jean-Pierre Goldenstein, Linguistique et discours littéraire (Paris: Larousse, coll. 'L', 1976), 25

5 Scherer, 51

6 Adam, 23

7 Ibid.

8 Nombreux sont les critiques qui ont remarqué le 'soin,' l"efficacité' du dramaturge. Le commentaire suivant est plutôt typique dans ce sens-là: 'Beaumarchais avait prévu avec beaucoup de soin, et même avec minutie, la mise en scène de toutes ses pièces,' J. Scherer, la Dramaturgie de Beaumarchais (Paris: Nizet 1970), 61-2

9 Guy Michaud, l'Oeuvre et ses techniques (Paris: Nizet 1957), 250

10 Ibid., 250-1

11 A.I, sc. 4, p. 186

12 A. III, sc. 9, p. 262

13 Beaumarchais, le Mariage de Figaro, mise en scène et commentaires de Jean Meyer (Paris: Seuil, coll. 'Mises en scène,' 1953), 49

14 Ibid., 55

15 A.I, sc. 4, p. 186

16 Dans le manuscrit $F$, nous lisons ces quelques répliques 'en marge et barrées au crayon' qui prouvent que Suzanne comprenait fort bien la situation initiale:

FIGARO: $\quad$ Tu ris, friponne!

SUZANNE: Au reste, mon ami, maintenant que vous savez tout, nous prendrons ce logement s'il vous agrée.

FIGARO: $\quad$ Il me déplaît fort. (Je vous jure).

SUZANNE: $\quad$ Et pourquoi?

FIGARO: Je n'en veux point.

SUZANNE: On dit une raison!

FIGARO: $\quad$ N'en parlons plus.

SUZANNE: Ce logement si commode! Et zeste, en deux pas. 
cf. le Mariage de Figaro, publié par J.B. Ratermanis, dans Studies on Voltaire and the Eighteenth Century, 63 (1968), 38.

17 A.I, sc. 2, p. 184

18 La 'politique' du Comte, Figaro la dissèque habilement à l'Acte III:

... feindre d'ignorer ce qu'on sait, de savoir tout ce qu'on ignore; d'entendre ce qu'on ne comprend pas, de ne pas ouïr ce qu'on entend; surtout de pouvoir au delà de ses forces; avoir souvent pour grand secret de cacher qu'il n'y en a point; s'enfermer pour tailler des plumes, et paraitre profond quand on n'est, comme on dit, que vide et creux; jouer bien ou mal un personnage, répandre des espions et pensionner des traîtres; amollir des cachets, intercepter des lettres, et tâcher d'ennoblir la pauvreté des moyens par l'importance des objets: voilà toute la politique, ou je meure! (A. III, sc. 5, p. 259)

Il s'agit, en un mot, d'intrigue' plutôt que de politique.

19 Christiane Mervaud, 'le Ruban de nuit de la comtesse,' dans la Revue d'Histoire littéraire de la France, 5 (septembre/octobre 1984) 727

20 A. III, sc. 4, p. 253; A. IV, sc. 5, p. 294

21 Scherer, 81

22 Ibid., 85

23 A. I, sc. 9, p. 201

24 De l'avis de J. Scherer, 'on n'est pas habitué à voir un grand d'Espagne écouter en se cachant; aussi le Comte doit-il, dans un aparté, dire au public qu'il écoute et pourquoi il écoute.'(91). La raison du Comte: 'Voyons un peu comme il me sert' (A. I, sc. 9, p. 197), n'arrive toutefois pas à l'excuser; elle confirme plutôt son incapacité à bien juger ses propres sujets.

25 Pour Ch. Mervaud, le ruban 'rapproche les cœurs, mais aussi les corps et leur permet de s'aimer par procuration.' (732). Selon nous, la présence de Chérubin dans le fauteuil et, plus tard, dans le cabinet de toilette de la Comtesse montre à quel point il a réussi à usurper - bien que ce ne soit que symbolique pour l'instant - la place du Comte. N'oublions pas que le page a précédé le Comte chez Fanchette, chez Suzanne, et qu'il le précédera, sous peu, dans le lit de la Comtesse.

26 A. I, sc. 9, p. 199

27 Ibid., p. 201

28 Scherer, 101

29 Pour Anne Ubersfeld, 'L'idée fondamentale du Mariage, c'est la revendication pour le tiers état, représenté par Figaro, de ses droits à l'existence et au bonheur. Et, une fois admise la revendication de l'intelligence, les droits politiques ne sont pas loin.' La scène où tous réclament l'abolition du droit du seigneur nous semble bien appuyer cette thèse. cf. Beaumarchais, le Mariage de Figaro, préface et notes d'Annie Ubersfeld (Paris: Editions Sociales 1968), 24-46

30 Scherer, 105

31 A. III, p. 252 
32 A. III, sc. 7, p. 261

33 A. III, sc. 15, p. 269

34 A. III, sc. 13 , p. 268

35 Scherer, 344 